\title{
तII UNIVERSITY of MARYLAND SCHOOL OF MEDICINE
}

\section{Outcomes of Tibiotalocalcaneal Hindfoot Fusion Nails in the Setting of Acute Lower Extremity Trauma}

Kalin J. Fisher MD, Steven F. Shannon MD, Christina M. Dean BS, Max A. Coale BS,

Robert V. O'Toole MD, Theodore T. Manson MD, Marcus F. Sciadini MD

${ }^{*}$ R Adams Cowley Shock Trauma Center, Department of Orthopaedics, University of Maryland School of Medicine, Baltimore, Maryland

\section{INTRODUCTION}

High energy fractures of the distal lower extremity in patients with significant medical comorbidities and lower functional demand present a difficult clinical challenge.

- Traditional surgical treatment with ORIF, either acutely or staged, has been associated with a high rate of wound complication, hardware failure, nonunion and/or infection in patients with multiple medical comorbidities.

- Non operative treatment may also lead to poor outcome due to fracture instability, skin issues with casting and potential for non-union and malunion.

- Due to the inherently poor soft-tissue envelope, the occurrence of any complication in a compromised host frequently results in amputation.

- In select, high-risk patients, acute hindfootfusion nailing through percutaneous incisions has been employed in an effort to mitigate the complication profile in a challenging patient population.

\section{HYPOTHESIS}

- The purpose was to determine the outcomes of high-risk patients receiving a tibiotalocalcaneal hindfoot fusion (TTC) nail in the setting of acute lower extremity trauma.

- We hypothesized that TTC nails would be associated with an acceptable rate of limb salvage and complication even in this group with severe comorbidities and at-risk fractures.

\section{METHODS}

- We performed a retrospective chart review of 54 adult patients with significant medical comorbidities receiving a TTC nail in the setting of an acute lower extremity fracture at a single level I trauma center between 2007 and 2016.

- Medical comorbidities included diabetes mellitus, cardiovascular disease, mental health diagnoses, renal disease, peripheral vascular disease, morbid obesity, peripheral neuropathy, extreme age related frailty, and low functional demand.

- Most patients had multiple comorbidities.

- Primary outcome measure was unexpected return to the operating room within two years after the index procedure.

\begin{tabular}{|l|l|}
\hline Table 12 & $\%(\mathrm{n})$ \\
\hline Open fractures & $543.7 \%(\mathrm{n}=29)$ \\
\hline Complete articular tibial pilon (43C1-3) & $50.0 \%(\mathrm{n}=27)$ \\
\hline Ankle fractures (44B/44C) & $22.2 \%(\mathrm{n}=12)$ \\
\hline Partial articular tibial pilon (43B1-3) & $11.1 \%(\mathrm{n}=6)$ \\
\hline Extraarticular tibial pilon (43A1-3) & $5.56 \%(\mathrm{n}=3)$ \\
\hline Distal diaphyseal tibia & $34.70 \%(\mathrm{n}=2)$ \\
\hline Talus fractures & $43.70 \%(\mathrm{n}=2)$ \\
\hline Charcot arthropathy & $43.70 \%(\mathrm{n}=2)$ \\
\hline Calcaneus fractures & $1.852 \%(\mathrm{n}=1)$ \\
\hline
\end{tabular}

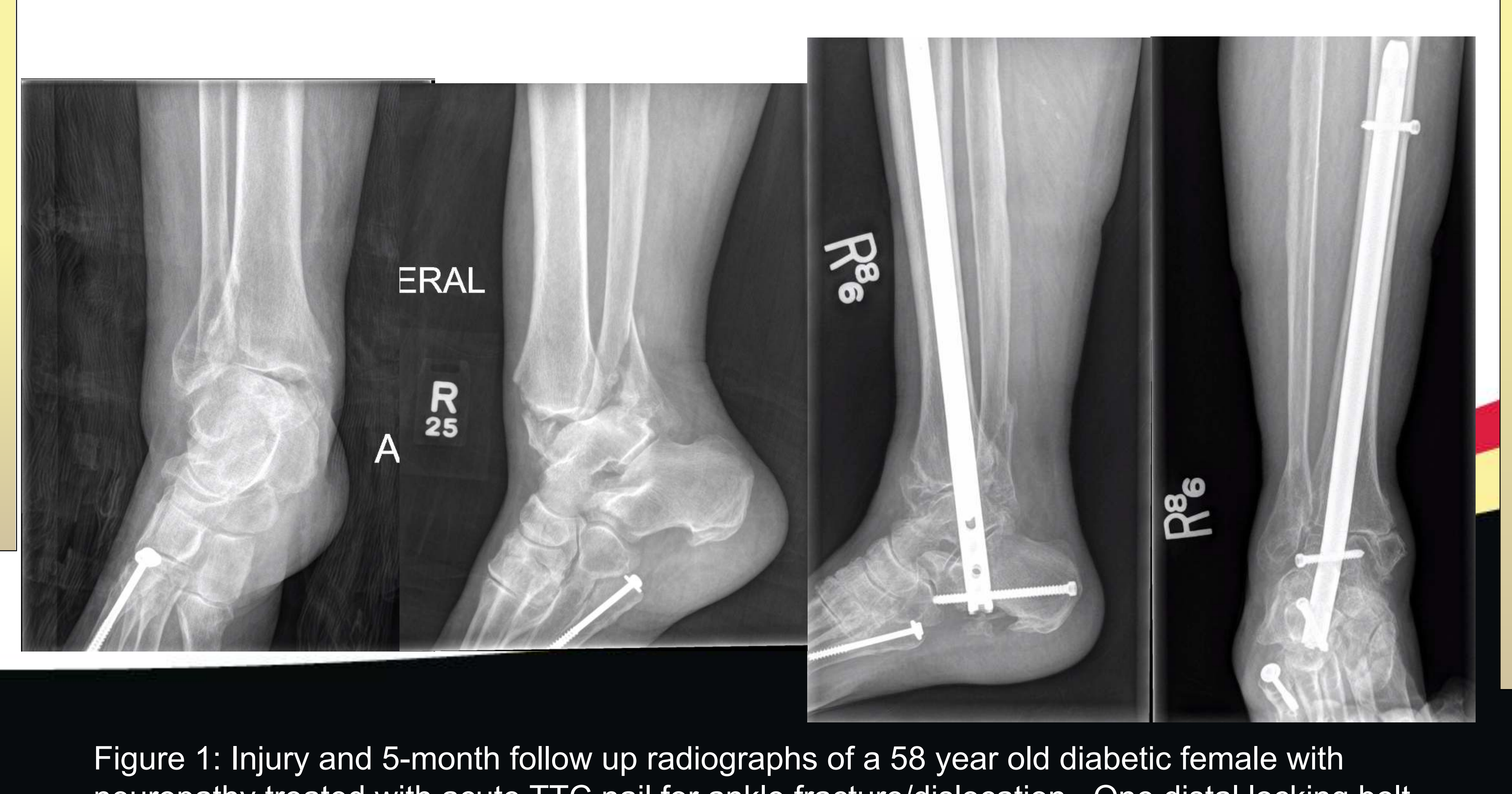

\section{RESULTS}

- Of 54 patients included in this study, 22 patients $(41 \%)$ had an unexpected return to the operating room (RTOR), with some patients requiring more than one follow up procedure.

- The most common reason for RTOR was hardware removal $(37 \%)$ followed by repeat irrigation and debridement (24\%).

- Four patients required revision TTC fusion: two for osteomyelitis, one patient for a symptomatic broken talar screw, and one patient for post-traumatic arthritis after nail removal.

- Only 4 patients (7\%) ultimately required amputation, all due to chronic osteomyelitis.

\section{CONCLUSIONS}

In contrast to our hypothesis, there was still a high rate of RTOR for the patients in this study as well as deep surgical site infection $(24 \%)$

- However, there was a relatively low rate of amputation (7\%) compared to other studies examining this difficult patient population

- In spite of the high complication rate reported here, the limb salvage rate was high.

Presumably, management of deep surgical site infection is facilitated by use of percutaneous techniques and intramedullary implants for the index surgical procedure.

This technique may offer a reasonable alternative for achieving limb salvage in a select group of medically-challenging patients with severe lower extremity injuries and significant medical comorbidities. 